\title{
Theoretical fitting characteristics of typical soft contact lens designs
}

\author{
AUTHORS: Anna Sulley BSc MCOptom ${ }^{1}$ \\ Kathrine Osborn-Lorenz OD MS ${ }^{2}$ \\ James Wolffsohn FCOptom PhD MBA ${ }^{3}$ \\ Graeme Young FCOptom MPhil PhD FCOptom DCLP ${ }^{4,3}$
}
1 Johnson \& Johnson Vision Care Companies, Wokingham, UK
2 Johnson \& Johnson Vision Care, Inc, Jacksonville, FL, USA,
3 Aston University, Birmingham, UK
4 Visioncare Research Ltd, Farnham, UK

\section{ACKNOWLEDGEMENTS}

This work was supported by a grant from Johnson \& Johnson Vision Care Companies, Johnson \& Johnson Medical Ltd. We thank Hannah King and Chris Hunt from Visioncare Research Ltd for assistance with the preparation of the manuscript. 


\section{Theoretical fitting characteristics of typical soft contact lens designs}

\section{ABSTRACT}

PURPOSE: To calculate theoretical fitting success rates for a range of typical soft contact lens designs using a mathematical model.

METHOD: A spreadsheet mathematical model was used to calculate fitting success rates for various soft contact lens designs. The soft lens designs were evaluated using ocular topography data collected from 163 subjects. The model calculated success rates based on acceptable edge strain falling within the range 0 to $6 \%$ and horizontal diameter overlap falling within the range 0.2 to $1.2 \mathrm{~mm}$. In the case of those lenses with multiple base curves (BCs), eyes unsuccessful with the steeper BC were tested with the flatter $\mathrm{BC}$ and aggregate success rates calculated. The calculations were based on typical, currently available, hydrogel and silicone hydrogel soft lens designs and allowed for appropriate oneye shrinkage (1.0-2.3\%). The theoretical results were compared with those from actual clinical trials.

RESULTS: The theoretical success rates for typical one-BC lenses ranged from $60.7 \%(95 \% \mathrm{Cl} 7.2 \%)$ to $90.2 \%(95 \% \mathrm{Cl} 3.7 \%)$. With typical two-BC designs, most combinations showed an increase in success rate by using a second $\mathrm{BC}($ e.g. $84.0 \% \rightarrow 90.2 \%)$. However, one of the two-BC combinations showed only a negligible increase with a second $\mathrm{BC}(72.4 \% \rightarrow 73.0 \%)$. For designs_with lower success rates, the greatest contributor to failure was inadequate lens diameter. For a given lens design, differences in shrinkage (i.e. on-eye bulk dehydration) had a significant effect on success rate. In comparison with historical clinical data, there was a positive correlation between the prevalence of small lens fittings and actual discomfort reports $(r=+0.95, P=<0.001)$. However, there was a poor correlation between theoretical and actual tight/loose fittings.

CONCLUSIONS: Mathematical modelling is a useful method for testing soft contact lens design combinations. The results suggest that judicious choice of additional fittings can expand the range of fitting success.

Keywords: soft contact lens, diameter, base curve radius, tightness, corneal coverage, success rate 


\section{INTRODUCTION}

Many of the important characteristics of soft contact lens performance can be anticipated from physical measurements, such as modulus, oxygen permeability, thickness, and coefficient of friction. Elastic modulus, coupled with thickness profile, gives some indication of the lens' handling characteristics and the likelihood of mechanical induced complications. ${ }^{1-3}$ Oxygen transmission can be correlated to openeye and closed-eye corneal swelling and limbal hyperemia. ${ }^{4}$ Recently, coefficient of friction has been related to comfort. ${ }^{5}$

Many clinical trials attempt to compare the clinical performance of different clinical designs. However, even with modest subject numbers, these are expensive, time-consuming and are only able to compare a limited number of lens designs. An obvious alternative to the clinical testing of soft lens fitting success is to use computer modelling which is routinely used in the commercial development of soft lens designs. A spreadsheet model has been described to evaluate effect of various aspects of soft lens parameters. 6,7

The main fitting characteristics governing the success of well-fitting soft contact lenses include tightness, and corneal coverage. ${ }^{8}$ The model estimates tightness by calculating the increase in lens circumference, or edge strain, when a given lens design is fitted to a given eye shape. It estimates corneal coverage by calculating how much the lens overlaps the cornea when forced to align with the given ocular topography. ${ }^{7}$ Lens centration, movement and edge alignment are also important lens fit characteristics, however, these are consequent on lens tightness and therefore not considered by the model.

The technique incorporates some obvious approximations which limit its accuracy but some effort has been made in this study to correlate the findings with actual clinical data.

The purpose of this project has been to use spreadsheet modelling to assess the theoretical fitting success of a wide range of currently available spherical soft contact lenses and to compare this with historical data from previous clinical trials.

\section{METHOD}

This computer modelling study estimated the theoretical fitting success rates for a range of soft lenses using population data from a previous study. ${ }^{9}$ The mathematical model calculated soft lens fit characteristics (tightness, corneal overlap) against pre-set acceptance criteria and determined whether given lenses on given eyes were acceptable. The theoretical data were compared with historical data from previous JJVCI clinical trials.

\section{$\underline{\text { Subject Database }}$}

The subject database comprised data from 163 eligible UK subjects who had been examined in a previous study. ${ }^{9}$ To be included in the database, subjects were required to have a spectacle cylinder $\leq 2.00 \mathrm{D}$ and no keratoconus or other severe corneal irregularity contraindicating lens wear.

Ocular topography data from only one eye per subject were used; this was the right eye data unless only the left eye data were available.

The ocular topography inputs were corneal apical radius, corneal asphericity (shape factor), corneal diameter and corneoscleral junction angle (Table 1). The corneal diameter was the true corneal 
diameter as opposed to the conventional clinical measurement of horizontal visible iris diameter (HVID) which underestimates the true diameter by approximately $1.5 \mathrm{~mm} \cdot{ }^{9,10}$

Table 1: $\quad$ Summary of ocular topography data and demographics $(\mathrm{N}=163)$

\begin{tabular}{|l|lccc|}
\hline Ocular variable & Mean (SD) & Median & \multicolumn{2}{c|}{ Range } \\
\hline Apical radius (mm) & $7.78(0.30)$ & 7.78 & 7.01 & 8.77 \\
\hline Shape Factor & $0.52(0.16)$ & 0.53 & -0.01 & 0.91 \\
\hline Corneal Diameter (mm) & $13.39(0.44)$ & 13.4 & 12.10 & 14.41 \\
\hline Corneoscleral Junction ( $\left.{ }^{\circ}\right)$ & $175.4(2.3)$ & 175.4 & 166.2 & 179.7 \\
\hline Age (years) & $37.7(15.6)$ & 15.6 & 18 & 65 \\
\hline Sphere Refraction (DS) & $-1.62(2.3)$ & -1.25 & +3.00 & -9.25 \\
\hline Cylinder Refraction (DC) & $-0.57(0.45)$ & -0.50 & 0.00 & -2.00 \\
\hline Ethnicity & White: $79 \%$; East Asian British: $18 \% ; 2 \%$ Mixed \\
\hline \multicolumn{5}{|c}{ Race } \\
\hline
\end{tabular}

\section{Study Lenses}

A range of 15 current representative spherical soft lens designs were tested (Table 2). The base curve (BC) and diameter values were taken from manufacturer's specifications. The shrinkage factors were calculated from measurements of lens diameter at room and eye temperatures using an established method. ${ }^{3,11}$ This represents the amount of change in BC and diameter when lenses are placed on the eye and raised to ocular surface temperature.

For the purposes of the theoretical analysis, it was assumed that the lens back surface design was monocurve and spherical.

These lens designs were based on currently available designs and therefore represent a cross-section of contemporary lenses. For the six lens brands currently available in two BCs, the aggregate overall success rate was calculated as well as the individual success rates. 
Table 2: Study CLs sorted by shrinkage factor (shaded cells indicate hydrogel CLs)

\begin{tabular}{|c|c|c|c|c|c|}
\hline $\begin{array}{c}\text { Lens } \\
\text { Code }\end{array}$ & $\begin{array}{c}\text { Daily } \\
\text { disposable/ } \\
\text { Reusable }\end{array}$ & $\begin{array}{c}\text { Water } \\
\text { content (\%) }\end{array}$ & $\begin{array}{c}\text { BC } \\
(\mathbf{m m})\end{array}$ & $\begin{array}{c}\text { Diameter } \\
(\mathbf{m m})\end{array}$ & $\begin{array}{c}\text { Shrinkage } \\
\text { Factor }\end{array}$ \\
\hline A & DD & 46 & $8.50,9.00$ & 14.2 & 0.9969 \\
\hline B & DD & 38 & $8.50,9.00$ & 14.3 & 0.9932 \\
\hline C & Reusable & 38 & $8.40,8.80$ & 14.0 & 0.9929 \\
\hline D & Reusable & 41 & $8.40,8.80$ & 14.0 & 0.9926 \\
\hline E & Reusable & 24 & $8.40,8.60$ & 13.8 & 0.9920 \\
\hline F & DD & 33 & 8.50 & 14.1 & 0.9904 \\
\hline G & Reusable & 36 & 8.60 & 14.0 & 0.9901 \\
\hline H & Reusable & 33 & 8.60 & 14.2 & 0.9870 \\
\hline I & DD & 56 & 8.60 & 14.1 & 0.9821 \\
\hline J & DD & 54 & 8.40 & 14.2 & 0.9790 \\
\hline K & Reusable & 48 & 8.60 & 14.0 & 0.9786 \\
\hline L & DD & 58 & $8.50,9.00$ & 14.2 & 0.9772 \\
\hline M & DD & 69 & 8.70 & 14.0 & 0.9756 \\
\hline N & DD & 78 & 8.60 & 14.2 & 0.9571 \\
\hline O & DD & 59 & 8.60 & 14.2 & 0.9508 \\
\hline & & & & & \\
\hline
\end{tabular}

\section{Lens Analysis}

A spreadsheet computer model was used to calculate the lens fit success rates of various soft lens designs. ${ }^{7}$ This updated version allows separate inputs for vertical and horizontal ocular topography. It allows calculations to be done for two lens designs and to give an overall success rate, for example, for a lens design incorporating two base curves.

The lens inputs include diameter, BC and shrinkage factor for the given lens material (Table 2). The 15 lens designs were analysed using the database of 163 eyes (Table 2).

For a given lens and eye, the model determined whether the lens provides an acceptable fit based on two characteristics: i) edge tightness (or strain) and ii) horizontal corneal overlap. The edge strain was averaged for horizontal and vertical meridians. The lens' overlap of the cornea was based on only the horizontal meridian and the horizontal cornea is invariably larger than the vertical. For a lens to be judged acceptable, it was required to show mean edge strain falling within the range 0 to $6 \%$ and horizontal corneal overlap falling within the range 0.2 to $1.2 \mathrm{~mm}$. These thresholds were estimated using historical clinical study data.

Success rates were based on the proportion of the 163 eyes showing as acceptable fit for tightness, diameter overlap and overall acceptance. The overall success rate, therefore, indicated the proportion of lens fittings that were acceptable for both diameter and tightness. In the case of those lenses with 
multiple BCs, those eyes not successfully fitted with the steeper BC, were tested with the flatter BC and the aggregate success rates calculated.

\section{$\underline{\text { Historical Clinical Data Comparison }}$}

In order to evaluate the reliability of the model, the theoretical results for specific lens types were compared with actual clinical data from historical unpublished study data. Key lens fit data were extracted from eight previous clinical studies. The previous studies involved a representative sample of ten of the reusable and daily disposable lens types analysed and sample sizes varied from 100 to 258 subjects per lens type. The following summary data relating to lens fitting results were extracted:

- Proportion of eyes reporting frequent/constant discomfort

- Proportion of eyes with non-optimal loose or tight fittings, i.e. Grade $-1 /-2$ or Grade $+1 /+2(-2$ to +2 scale, 0-optimum), respectively.

\section{Statistical Analysis}

The 95\% confidence intervals were calculated for all success rates using the following formula: $\operatorname{SQRT}\left(\left(p^{*}(1-p)\right) / N\right)^{*} 1.96$ (where $p=$ proportion, $N=$ sample size)

Spearman's rank correlation coefficient was used to test for associations between selected variables.

\section{RESULTS}

\section{Lens Analysis}

The results are summarised in Table 3, and in Figure 1 (overall success rates) and Figure 2 (multiple BCs).

For the single $B C$ designs, the overall success rate ranged from $60.7 \%$ (Lens $M \& O$ ) to $90.2 \%$ (Lens $F \& H)$. The ranges were similar for single $B C$ design daily disposable $(60.7-90.2 \%$, Lens $M \& O$ and Lens $\mathrm{F}$ respectively) and for reusable lenses $(68.7-90.2 \%$, Lens $\mathrm{K}$ and Lens $\mathrm{H}$ respectively) (Table 3 , Figure 1).

Six of the lens types comprised two BCs. In each case, the steeper BC was the most successful overall. The use of an additional flatter BC increased the success rate, on average by $6 \%$ (median). The greatest increase was for Lens B for which the combined success rate was $95.1 \%$ compared with $82.22 \%$ for the steep BC only. The least successful combination was Lens $E$ for which the combined success rate was $73.0 \%$ compared with $72.4 \%$ for the steep BC only. With two BCs, high success rates were achieved with both daily disposable and reusable lenses (Table 3, Figure 2).

The highest overall success rate was, therefore, achieved by the two-BC combination of daily disposable silicone hydrogel Lens A $(95.7 \%)$. With reusables, the highest overall success rate was with Lenses C \& D (two BC) and Lens $F$ (one BC), all silicone hydrogel lenses (90.2\%).

Across all lens types, the most common reason for failure was excessively small diameter (19.7\%); lenses were found to be too large in only a small proportion of cases $(0.7 \%)$. Loose fittings were more common than tight fittings (5.4\% vs. $3.0 \%)$ (Table 3 ). 
Table 3: Summary of theoretical CL fit success rate calculations. Diameter success based on overlap criteria, tightness success based on edge strain criteria and overall success based on aggregate of both. (St: steep BC; FI: flat BC)

\begin{tabular}{|c|ccc|ccc|cc|}
\hline Lens & $\begin{array}{c}\text { Diameter } \\
\text { Success }\end{array}$ & Small & Large & $\begin{array}{c}\text { Tightness } \\
\text { Success }\end{array}$ & Tight & Loose & $\begin{array}{c}\text { Overall } \\
\text { success }\end{array}$ & $95 \% \mathrm{Cl}$ \\
\hline A-2BC & $95.7 \%$ & $4.3 \%$ & $0.0 \%$ & $96.3 \%$ & $0.0 \%$ & $3.7 \%$ & $95.7 \%$ & $2.2 \%$ \\
A-St & $92.6 \%$ & $3.7 \%$ & $3.7 \%$ & $93.9 \%$ & $4.9 \%$ & $1.2 \%$ & $89.0 \%$ & $3.9 \%$ \\
A-FI & $84.0 \%$ & $16.0 \%$ & $0.0 \%$ & $77.9 \%$ & $0.0 \%$ & $22.1 \%$ & $70.6 \%$ & $6.5 \%$ \\
\hline B-2BC & $95.1 \%$ & $4.3 \%$ & $0.6 \%$ & $96.9 \%$ & $0.0 \%$ & $3.1 \%$ & $95.1 \%$ & $2.4 \%$ \\
\hline B-St & $91.4 \%$ & $3.7 \%$ & $4.9 \%$ & $87.7 \%$ & $11.0 \%$ & $1.2 \%$ & $82.2 \%$ & $5.1 \%$ \\
B-FI & $89.0 \%$ & $10.4 \%$ & $0.6 \%$ & $79.8 \%$ & $0.0 \%$ & $20.2 \%$ & $74.8 \%$ & $6.0 \%$ \\
\hline C-2BC & $90.2 \%$ & $9.8 \%$ & $0.0 \%$ & $95.7 \%$ & $0.0 \%$ & $4.3 \%$ & $90.2 \%$ & $3.7 \%$ \\
\hline C-St & $90.2 \%$ & $9.2 \%$ & $0.6 \%$ & $92.0 \%$ & $6.7 \%$ & $1.2 \%$ & $84.0 \%$ & $4.8 \%$ \\
\hline C-FI & $73.0 \%$ & $27.0 \%$ & $0.0 \%$ & $89.6 \%$ & $0.0 \%$ & $10.4 \%$ & $69.9 \%$ & $6.5 \%$ \\
\hline D-2BC & $90.2 \%$ & $9.8 \%$ & $0.0 \%$ & $95.7 \%$ & $0.0 \%$ & $4.3 \%$ & $90.2 \%$ & $3.7 \%$ \\
\hline D-St & $90.2 \%$ & $9.2 \%$ & $0.6 \%$ & $92.0 \%$ & $6.7 \%$ & $1.2 \%$ & $84.0 \%$ & $4.8 \%$ \\
\hline D-FI & $73.0 \%$ & $27.0 \%$ & $0.0 \%$ & $89.6 \%$ & $0.0 \%$ & $10.4 \%$ & $69.9 \%$ & $6.5 \%$ \\
\hline E-2BC & $73.0 \%$ & $27.0 \%$ & $0.0 \%$ & $95.1 \%$ & $0.0 \%$ & $4.9 \%$ & $73.0 \%$ & $6.2 \%$ \\
\hline E-St & $73.0 \%$ & $27.0 \%$ & $0.0 \%$ & $97.5 \%$ & $0.6 \%$ & $1.8 \%$ & $72.4 \%$ & $6.3 \%$ \\
\hline E-FI & $62.6 \%$ & $37.4 \%$ & $0.0 \%$ & $94.5 \%$ & $0.0 \%$ & $5.5 \%$ & $62.6 \%$ & $7.1 \%$ \\
\hline F & $91.4 \%$ & $8.0 \%$ & $0.6 \%$ & $96.9 \%$ & $1.2 \%$ & $1.8 \%$ & $90.2 \%$ & $3.7 \%$ \\
\hline G & $78.5 \%$ & $21.5 \%$ & $0.0 \%$ & $95.1 \%$ & $0.6 \%$ & $4.3 \%$ & $77.9 \%$ & $5.7 \%$ \\
\hline H & $91.4 \%$ & $8.0 \%$ & $0.6 \%$ & $97.5 \%$ & $1.2 \%$ & $1.2 \%$ & $90.2 \%$ & $3.7 \%$ \\
\hline I & $79.8 \%$ & $20.2 \%$ & $0.0 \%$ & $98.2 \%$ & $0.6 \%$ & $1.2 \%$ & $79.1 \%$ & $5.5 \%$ \\
\hline J & $90.8 \%$ & $7.4 \%$ & $1.8 \%$ & $81.6 \%$ & $18.4 \%$ & $0.0 \%$ & $73.6 \%$ & $6.2 \%$ \\
\hline K & $69.3 \%$ & $30.7 \%$ & $0.0 \%$ & $95.1 \%$ & $0.6 \%$ & $4.3 \%$ & $68.7 \%$ & $6.6 \%$ \\
\hline L & $90.2 \%$ & $9.2 \%$ & $0.6 \%$ & $92.0 \%$ & $6.7 \%$ & $1.2 \%$ & $84.0 \%$ & $4.8 \%$ \\
\hline M & $62.0 \%$ & $38.0 \%$ & $0.0 \%$ & $92.6 \%$ & $0.6 \%$ & $6.7 \%$ & $60.7 \%$ & $7.2 \%$ \\
\hline N & $69.3 \%$ & $30.7 \%$ & $0.0 \%$ & $98.2 \%$ & $1.2 \%$ & $0.6 \%$ & $68.1 \%$ & $6.7 \%$ \\
\hline
\end{tabular}


Figure 1: Summary of overall success rates for representative CL designs. Only steepest base curve (BC) shown where multiple BCs available (Dark shaded bars indicate hydrogel designs, spotted bars indicate daily disposables, error bars indicate $95 \%$ confidence intervals)

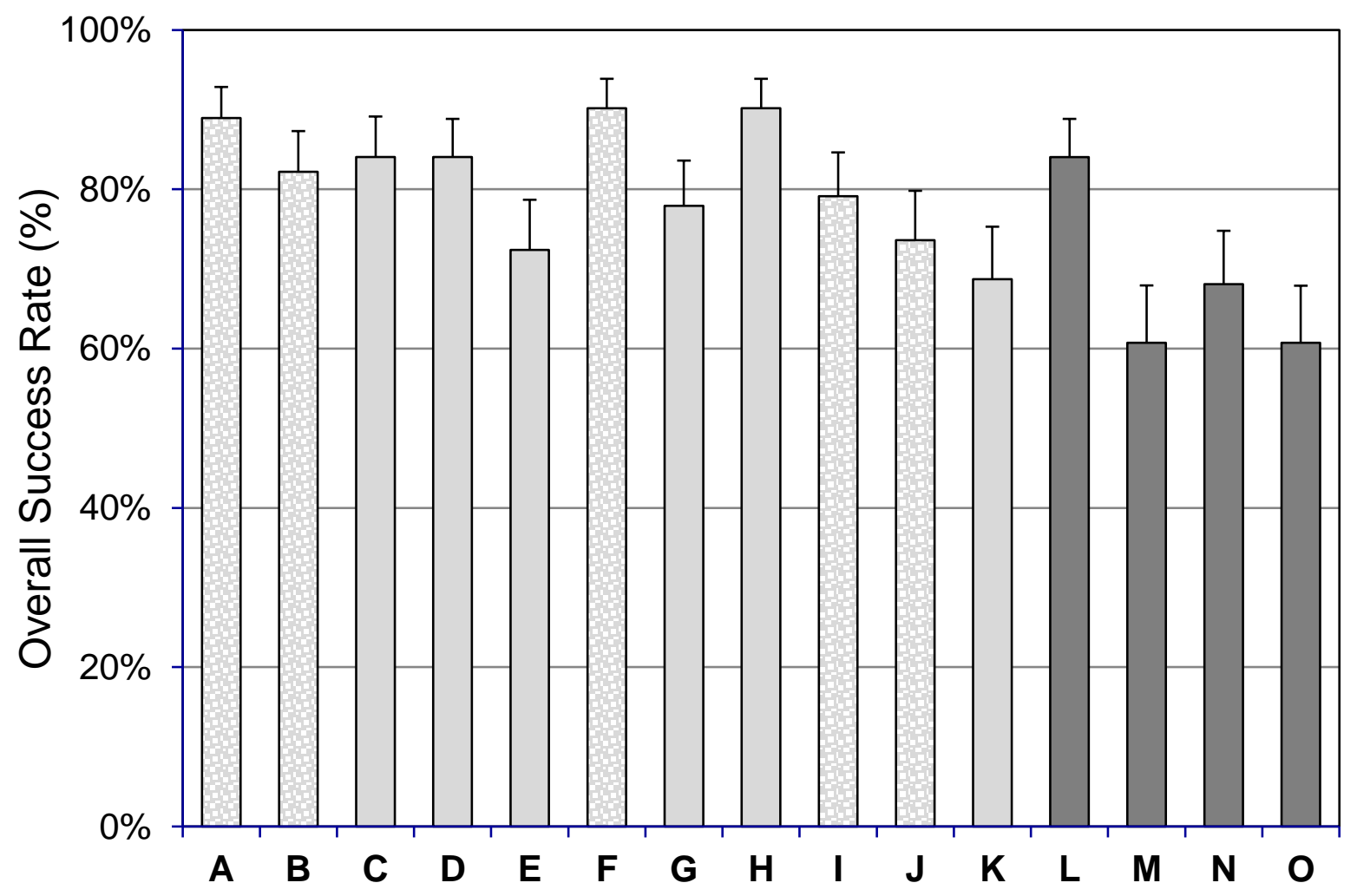


Figure 2: Summary of overall success rates (SRs) for multiple base curve (BC) designs. (Textured bars indicate aggregate SRs for two BCs. Error bars indicate $95 \%$ confidence intervals; St steep: BC; FI: flat BC)

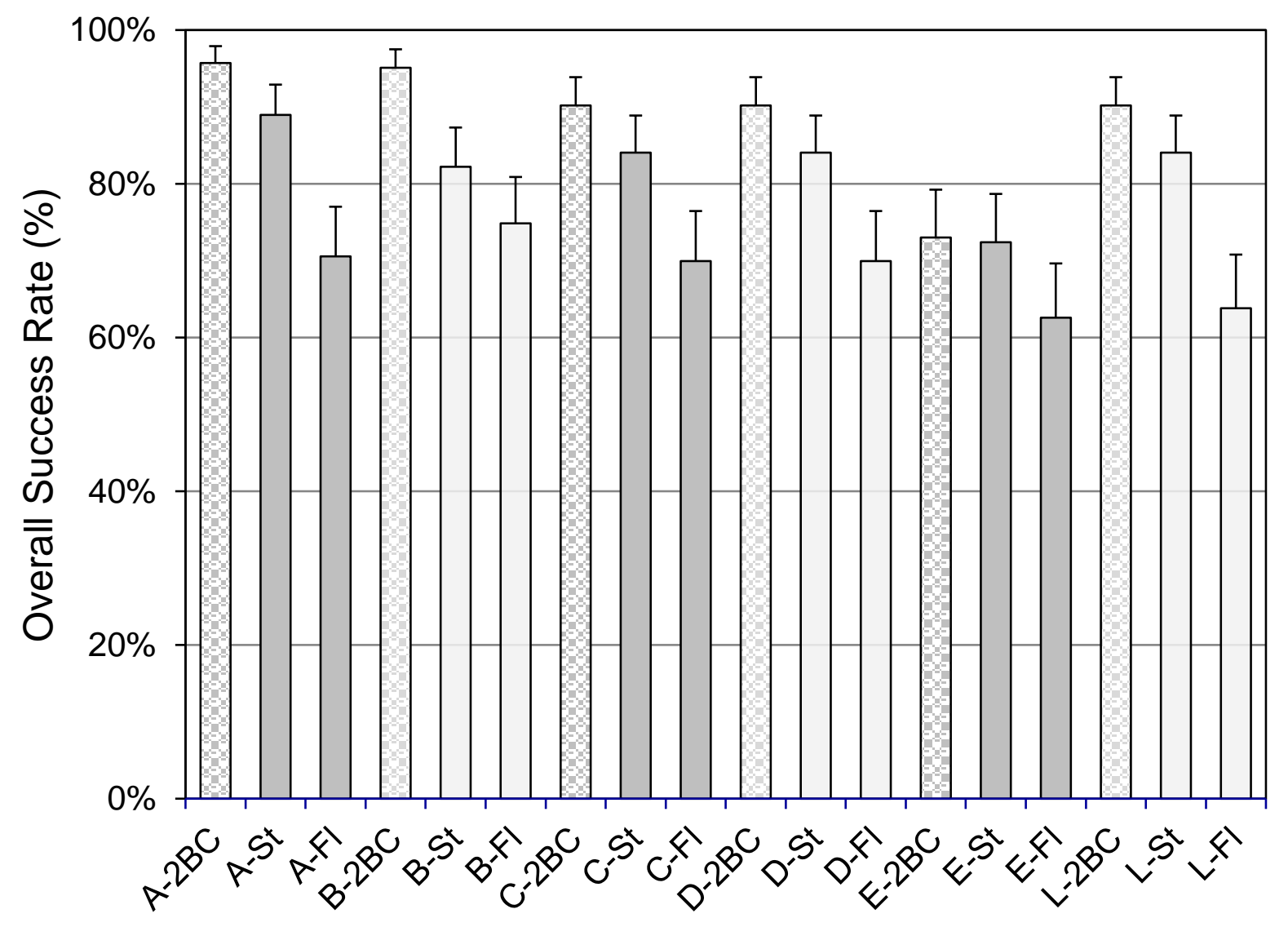

Historical Clinical Data Comparison

Actual historical clinical data were used to assess the validity of the computer model estimations. In order to assess the validity of the most common reason for failure (too small), the prevalence of theoretically small fittings was compared with the prevalence of uncomfortable fittings (Figure 3, Table 3). Clinical data for ten of the lens types in the analysis were used and compared against the theoretical data. There was a significant positive correlation between the prevalences of small fittings and reported discomfort $(r=+0.95, P<0.001)$. In other words, lenses with a high prevalence of theoretically small fittings tended to be less comfortable. 
Figure 3: Comparison of prevalence of uncomfortable fittings versus theoretical prevalence of small fittings ( $n=100$ to 258 subjects per sample)

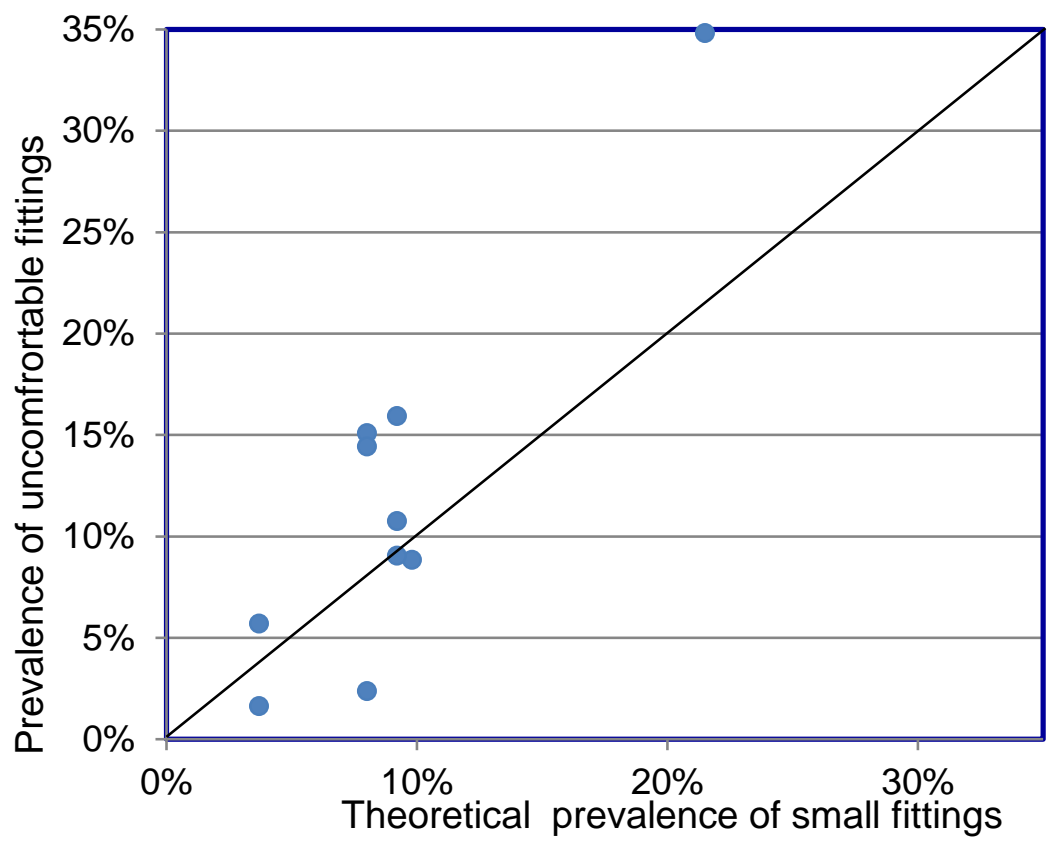

In order to assess the validity of the tightness assessments, the prevalences of actual sub-optimal fittings were compared with the theoretical prevalences of tight and loose fittings (Figure 4). Clinical data for ten different lens types were used and compared against the theoretical data. With the tight lens fittings, those lenses showing a higher proportion of theoretical tight fitting also tended to show a higher proportion of actual tight fittings, however, the correlation was not statistically significant $(r=+0.42, P=0.31)$. There was no apparent trend with loose fittings. 
Figure 4: Comparison of the prevalence of actual sub-optimal fittings (tight \& loose) versus theoretical prevalences ( $n=100$ to 258 subjects per sample)

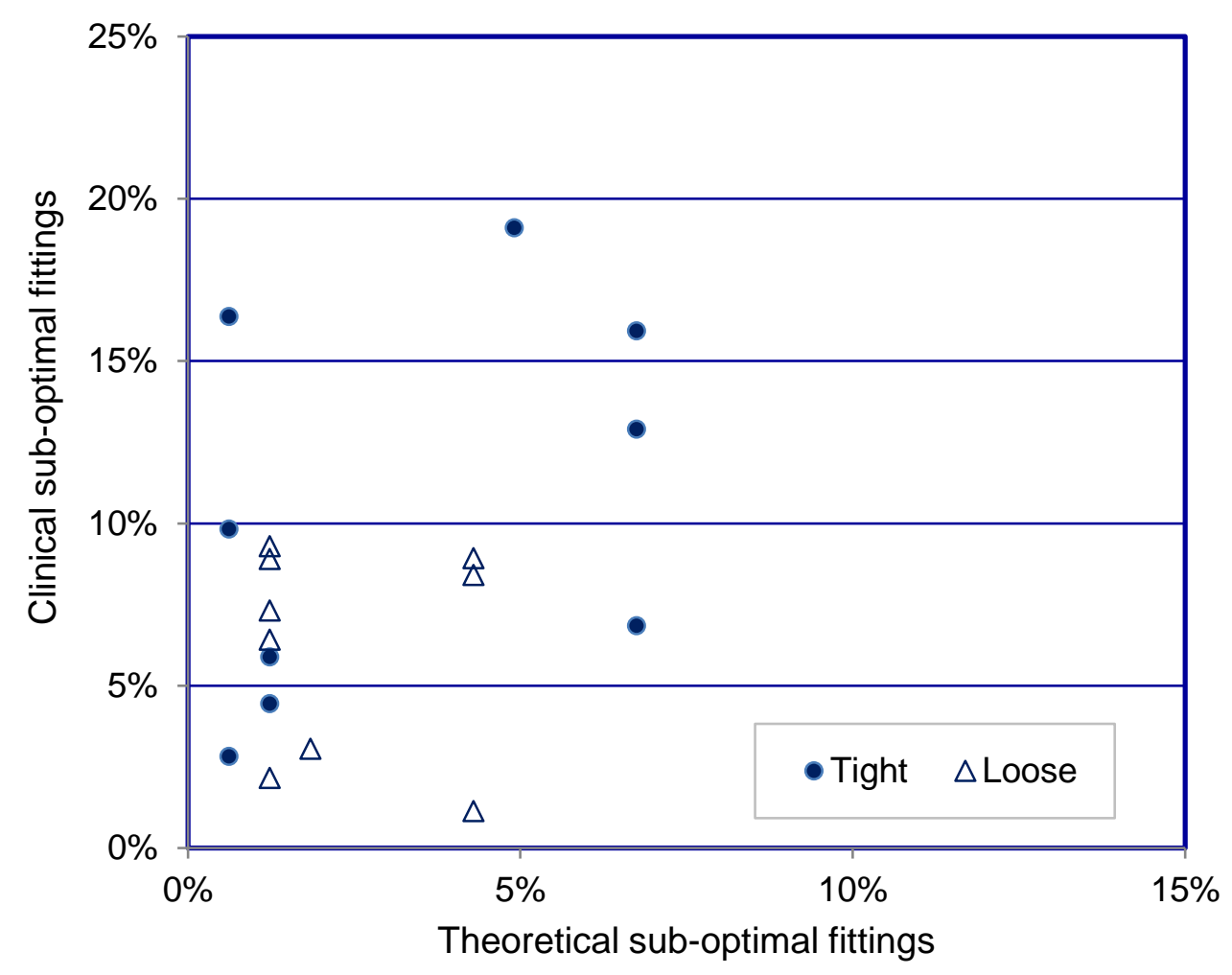

\section{DISCUSSION}

Commercially available soft CLs tend to lie within a narrow range of BCs and diameters. The lenses assessed in this study were within a relatively narrow range of parameters: labelled BCs between 8.40 and $9.00 \mathrm{~mm}$ and diameters between 13.8 and $14.3 \mathrm{~mm}$.

Despite the seemingly narrow parameter ranges, theoretical fit success rates varied widely between lens types (60.7-95.7\%). The reason for this wide variation is twofold. First, the large variation in shrinkage factor results in a wide variation in on-eye diameters. ${ }^{4}$ After allowing for shrinkage at eye temperature, the range is $13.5-14.2 \mathrm{~mm}$. Second, the combination of BC and diameter is also important; lenses at the flatter end of the range are less successful when combined with a relatively small diameter as this results in design of relatively small surface area and, therefore, small on-eye diameter.

In the case of the lenses achieving the poorest success rate (Lens $M \& O, 60.7 \%$ ), both were daily disposable, single BC designs. Reusable single BC design success rates ranged from $68.7 \%$ (Lens $\mathrm{K}$ ) to $90.2 \%$ (Lens $\mathrm{H}$ ). The poorer success rates are consistent with previous studies evaluating a wide range of lens designs. ${ }^{12-14}$ However, they might seem lower than typical manufacturer claims of success. The discrepancy might be explained by those fittings that seem acceptable at dispensing but 
later prove to be uncomfortable due to inadequate corneal coverage. Discomfort is frequently cited as a reason for the relatively high discontinuation rate from soft contact lenses. ${ }^{15-16}$

The use of two BCs increased the overall success rate with each of the six lens types; however, this increase varied by lens brand. The second $B C$ increased the overall success rate by $6 \%$, on average, but in all but one case, the combined success rate was $90 \%$ or more. Lens A achieved an impressive combined success rate of $95.7 \%$. It is interesting that, with Lens $E$, the flatter BC only facilitated a minimal improvement in success rate $(0.6 \%)$.

The proportion of theoretical lens fittings found to be tight or loose was relatively small and reflects the situation found in clinical practice. It is notable that inadequate corneal coverage was the most common reason for potential failure. Even with the most successful design (Lens $\mathrm{F}$ ), $8 \%$ of fittings were calculated as being too small. This suggests that, for most lens types, the greatest opportunity lies in providing additional designs that give a larger on-eye diameter.

One of the most striking findings of the study is the suggestion that a high proportion of lens fittings fail to give adequate corneal coverage and that this is the most common reason for lens failure. This prompts the question as to why this is not more commonly recognised; the answer may lie in the fact that true corneal diameter is typically $1.5 \mathrm{~mm}$ larger than the horizontal visible iris diameter (identified in clinical practice) and that this shortcoming is not always obvious to practitioners when viewing with a slit lamp. The most likely effect of inadequate corneal coverage is discomfort from pressure of the lens edge as it impinges onto the cornea, and this may be the case. Those lens types that are relatively small on the eye have also tended, in previous studies (Figure 3), to result in a high proportion of uncomfortable lens fits. Since it is known that other factors, such as surface lubricity or edge design, affect soft lens comfort, this finding is particularly compelling.

These comparisons of the theoretical fit data with historical clinical data provide some support for the validity of the model, although do not confirm it. The tight lens fittings appear to show a trend of studies finding a greater proportion of tighter fittings with those lens types predicted by the model to show a higher proportion. However, perhaps because of the small sample size, the correlation was not statistically significant.

\section{Limitations}

Inevitably with mathematical modelling, there are limitations to the methodology. Some of these have been described elsewhere. ${ }^{6,7}$

In assumed order of descending importance, these include the following: i) the theoretical fit calculations are based on labelled, manufacturer values, however, with some lens types, the actual measurements may differ from the labelled values ${ }^{17}$; ii) the calculations assume that each lens has a spherical, monocurve back surface profile; iii) the model makes no allowance for variation in modulus; iv) the model assumes no decentration; v) the model assumes that the lenses align with the front surface of the eye. The most notable limitation is the lack of allowance for modulus; it is likely that high modulus materials have a lower tightness threshold and, therefore, slightly poorer success rate. Decentration can have a critical effect on corneal coverage, however, experience suggests that large amounts of decentration either accompany a fitting which is also excessively loose or where conjunctival anomalies such as pingueculae prevent normal centration. 


\section{CONCLUSIONS}

Mathematical modelling allows rapid evaluation of varying soft $C L$ designs and design combinations on a large population sample.

Despite the narrow range of lens parameters, the theoretical success rates showed wide variation (61$90 \%$ ), for two reasons: i) large variation in shrinkage factor, and ii) importance of selecting the right combination of $\mathrm{BC}$ and diameter.

The use of a second $\mathrm{BC}$ increased the overall success rate; typically, a second, flatter $\mathrm{BC}$ increased the success rate by $7 \%$ suggesting that judicious choice of additional fittings can expand the range of fitting success.

\section{REFERENCES}

1. Lin MC, Yeh TN. Mechanical complications induced by silicone hydrogel contact lenses. Eye Contact Lens. 2013; 39:115-24.

2. Ozkan J, Mandathara P, Krishna P, Sankaridurg P, Naduvilath T, Willcox MD, Holden B. Risk factors for corneal inflammatory and mechanical events with extended wear silicone hydrogel contact lenses. Optom Vis Sci. 2010; 87:847-53.

3. Young G, Garafalo R, Peters S, Harmer CO. The effect of temperature on soft contact lens modulus and diameter. Eye Cont Lens 2011; 37: 337-341.

4. Morgan PB, Brennan NA, Maldonado-Codina C, Quhill W, Rashid K, Efron N. Central and peripheral oxygen transmissibility thresholds to avoid corneal swelling during open eye soft contact lens wear. J Biomed Mater Res B Appl Biomater. 2010; 92:361-5.

5. Jones L, Brennan N, Gonzalez-Meijome J, Lally J, Maldonado-Codina C, Schmidt T, Subbaraman L, Young G, Nichols J. The international workshop on contact lens discomfort: Report of the contact lens materials, design \& care subcommittee. Inv Ophthal Vis Sci 2013; 54: TFOS37-70.

6. Young G. Mathematical model for evaluating soft contact lens fit. Optom Vis Sci 2014; 91:167176.

7. Young G, Sulley A, Osborn K, Hall L, Wolffsohn J. The inter-relationship of soft contact lens diameter, base curve radius, and fit. Optom Vis Sci In press.

8. Young G. Evaluation of Soft Contact Lens Fitting Characteristics. Optom Vis Sci 1996; 73(4):247-254.

9. Hall L, Hunt C, Young G, Wolffsohn J. Factors affecting corneoscleral topography. Invest Ophthal Vis Opt 2013; 54:3691-3701.

10. Martin DK, Holden BA. A new method for measuring the diameter of the in vivo human cornea. Am J Optom Physiol Opt 1982; 59:436-441.

11. Young G, Potts M, Sulley A. The effect of temperature on soft contact lens diameter. Eye Cont Lens 2016;42:298-302.

12. Wake E, Tienda JB, Uyekawa PM, Mandell RB. Centration and coverage of hydrogel contact lenses. Am J Optom Physiol Opt. 1981; 58:302-8. 
13. Young G, Allsopp G, Inglis A, Watson S. Comparative performance of disposable soft contact lenses. Contact Lens and Anterior Eye 1997; 20:13-21.

14. Young G, Holden B, Cooke G. The influence of soft contact lens design on clinical performance. Optom Vis Sci 1993; 70:394-403.

15. Pritchard N, Fonn D, Brazeau D. Discontinuation of contact lens wear: a survey. Int Contact Lens Clin. 1999; 26:157-162.

16. Richdale K, Sinnott LT, Skadahl E, Nichols JJ. Frequency of and factors associated with contact lens dissatisfaction and discontinuation. Cornea 2007; 26(2):168-74.

17. Ozkan J, Ehrmann K, Meadows D, Lally J, Holden B, de la Jara PL. Lens parameter changes under in vitro and ex vivo conditions and their effect on the conjunctiva. Cont Lens Anterior Eye. 2013; 36:171-5. 